\title{
Fuzzy Classification Strategy for the Hole of Incomplete Mass Point Clouds of Irregular Model
}

\author{
Liu Yan-zhong ${ }^{1}$, LiuYan-ju ${ }^{2}$, Li Cheng ${ }^{1}$ and Zhang Hong-lie ${ }^{1}$ \\ ${ }^{1}$ Communication and Electronic Engineering Institute, Qiqihar University, \\ Qiqihar, China \\ ${ }^{2}$ Computing Center, Qiqihar University, Qiqihar, China \\ *15146692464@163.com
}

\begin{abstract}
This paper presents fuzzy classification strategy for the hole-filling that can classify the incomplete mass point clouds and improve the precision. The irregular model is complex object that some part is smooth and some parts are irregular including sharp features. Therefore, we put kNN and curvature of mass point clouds to fuzzy inference system to divide the type of the hole of mass point clouds and the output of FIS can determine which part of point clouds belong to. For different kind holes, corresponding algorithm is given. Point clouds in the smooth area are reconstructed by implicit directly and ones in other regions of thin or sharp area are reconstructed by attach points. This method is simpler than those complex methods used on the whole point clouds directly. The experiment results show that classification can save much time and surface reconstruction is very fine.
\end{abstract}

Keywords: mass point clouds; hole-filling; fuzzy inference; classification strategy; implicit function

\section{Introduction}

The hole in the three-dimensional reconstruction is a very common and inevitable problem. Mass point clouds obtained by the model of 3D scanner are incomplete, because some deep part of the model cannot be measured by the scanner. In addition, some problems, such as the error of the model and scanning position are inevitable, there be exist the hole of point clouds even by multi-angle scan and data fitting. In recent years, repairing the hole has been an important issue in the reconstruction of 3D model. Many scholars proposed their repairing methods in the 3D reconstruction. Most methods assumed that the missing data together with the geometry information does not exist and extracted this information from the shape of the model around the hole. These techniques of hole repairing can be roughly divided into two classes: one based on the volume rendering and the other based on surface rendering.

For the volume rendering algorithm, the model or sampling data is usually included in the volume of the grid, fill all the holes at the same time in the reconstruction of the whole shape [1]. There are some algorithms, such as splitting-merging method [2], encryption algorithm [3], the shell triangulation algorithm [4] and two-step [5]. This kind of method is usually use a regular grid or adaptive grid. Because this kind of method are built by basic mobile cube and has robustness, small holes and high resolution model can be conducted, even some geometry form of grid division can be repaired the corresponding holes also. But these methods cannot allow to choice of management and repair completely according to the idea of customers.

For the surface rendering algorithm, information is derived from the geometric analysis of the model. Bendels et al. [6] and Tekumalla et al. [7] extended image text repair synthesis method, found similar to the hole boundary surface around the hole by the 
analysis of the model, and then repaired the hole by the existing surface [8], which could get very good repairing effect and choose a hole repair conveniently. But the more edges or boundary of the hole was, the higher the complexity of the algorithm was. Parus and Kolingerova [9] put forward a simplified algorithm that can repair a hole at a moment and deduce the new information from the analysis of hole near the edge of surface shape. Carr et al. [10], Amenta and Kil [11] made smooth about repairing surface respectively by $\mathrm{RBF}$ and moving least squares method. Liepa [12] proposed to connect to triangular polygon and fill the hole directly, and then the segmentation and edge exchange way to eliminate the bad shape new triangles, and smoothing processing at last. Although the effect is good, the method needs to fill in length and angles of the relation between the triangles to generate new triangles and result in unnecessary waste of CPU.

The idea of the iteration is derived from the data around the lost information. Przybilla [13] got the data from the photo image and repaired the holes on the building model. But it was very troublesome that had two kinds of different nature of the integrated application of information. Xu et al. [14] proposed a very simplified data source to put forward that a photograph of an object was used to estimate the normal vector sets and could produce a smooth surface of cover on the real object shape. But it did not give a concrete example. Similar Matteo Dellepiane [15] proposed that digital camera was used to obtain one or more color images including scanning data information. However, the algorithm was very large in time consumption during geometric model of the object derived from the pixel information. Zhao wei [16] proposed method of triangular mesh to repair the hole. It expected the normal vector calculation scheme for the newly established the normal vector of triangular grid and finally each new vertex three-dimensional coordinates were replaced by poisson equation of the normal vector. For repairing of complex holes, mature complicated classic algorithms has not used yet. On the whole, the existing algorithm of hole repairing can only achieve a smooth surface such as dental crowns repair, as shown in figure 1. For model large and irregular curvature changes, there is no the issue to solve in field of repairing hole.
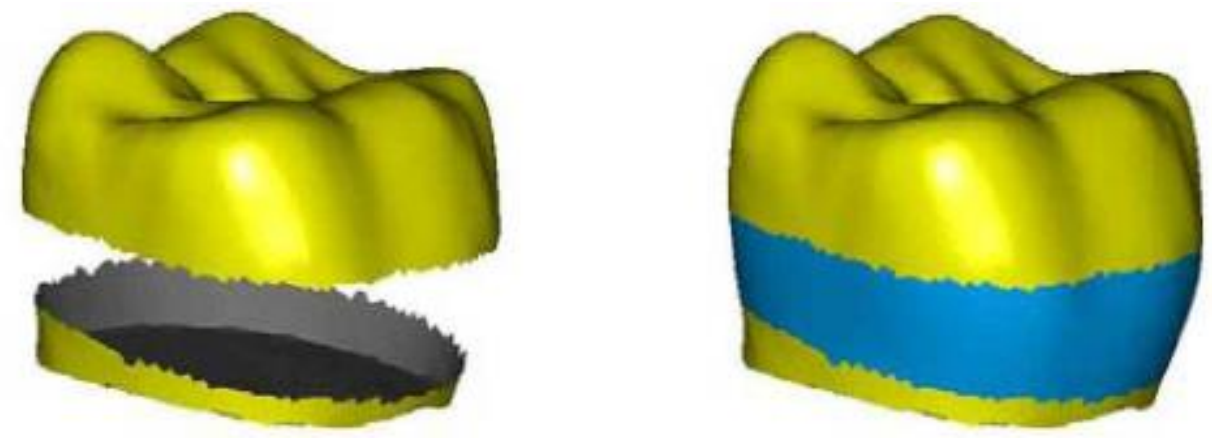

\section{Figure 1. Denture Repairation}

In this paper, we propose a novel fuzzy inference frame of classification strategy for the hole of incomplete point cloud of irregular model. The algorithm includes data mining for the incomplete point cloud existed, deducing the association rules for parameters of these data, determine the set of fuzzy rules to classify the type of hole constructed by point clouds and determine the change trend of unknown point cloud by the position relationship between local and global model entities. And design the fuzzy estimation model to compute the new data and parameters of data.

The paper is organized as follows. The related works of point clouds classification are reviewed in Section 2. In Section 3, the type of hole for incomplete point clouds are classified by Bayesian classifier. The classifier can restore feature of surface. Three cases of mesh mergence are proposed in Section 4. Conclusions are given in Section 5. 


\section{Storage of Mass Point Clouds}

Mass point clouds are stored in text file before using and data are read to Point_3D structure firstly.

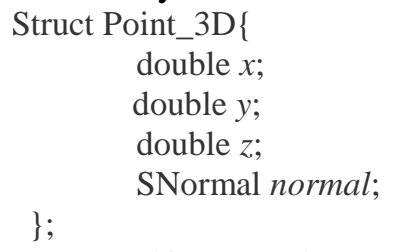

where normal is normal vector of point, if the text file do not exist the normal of data, the normal can be ascribed zero. In order to improve the precision of surface reconstruction, no matter normal value should be in the section 4 to this data to estimate. Each point of the corresponding values Morton in Morton_3D structure is stored by Morton sequence generation algorithm.

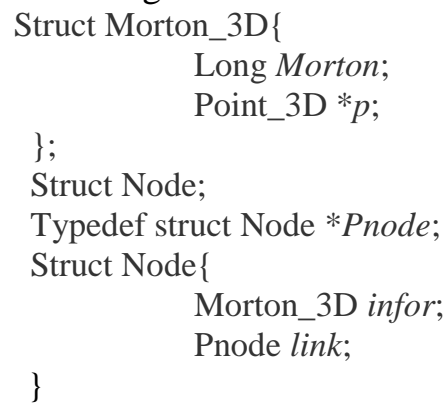

Morton sequence curve can divide add cube for the two-dimensional square, and then to sort these squares until each contain more than one point in the square using recursive partitioning approach. Quadtree, octree or other data structure are used to traversal point data in the $2 \mathrm{D}$ and $3 \mathrm{D}$.

\section{Characters of Mass Point Clouds}

Amplify the point clouds analysis, it can be seen in figure 2 that the density of point clouds is uncertainty. The left side of the point clouds distributes loose and K-nearest distance of center point is large between adjacent points. On the contrary, the right side of the data distribution is so dense the sum for the k-nearest distance is smaller. The point clouds can be seen their curvature changes small and local curved surface is smooth on the left side in figure 3 while curvature of the point clouds changes frequently on the right side. There's no rule to form local curved surface with sharp features, if only one times between smooth surfaces containing thin slices of the change of curvature is surface characteristics.
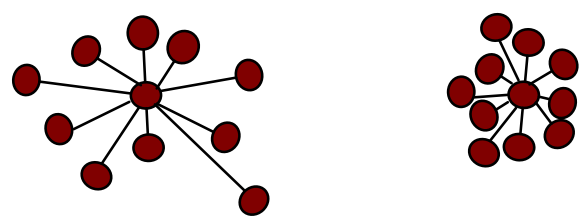

Figure 2. Distribution of Point Clouds

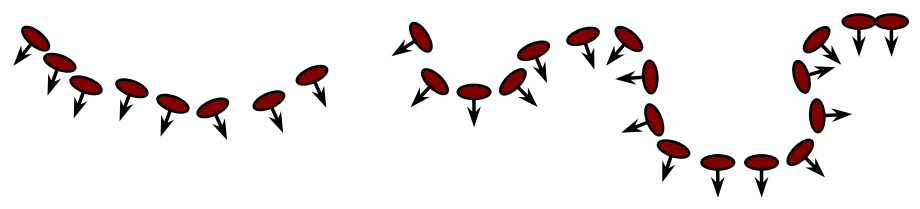

Figure 3. Curvature of Point Clouds 
Point clouds of the k-nearest distance and the combination of the curvature can represent the model characteristics of the region.

\section{Fuzzy Classification Strategy}

Because distribution of the point clouds has no law, it is necessary to introduce people experience to classify the point and repair the hole of mass point clouds.

\subsection{Fuzzy Inference}

The fuzzy rule base consists of a collection of fuzzy IF-THEN rules:

$$
R_{j} \text { : if } x_{k n n} \text { is } A_{1}^{j} \text { and } x_{\text {cur }} \text { is } A_{2}^{j} \text {, then } y \text { is } B_{m}
$$

where $\mathrm{x}={ }^{\left[x_{k n n}, x_{c u r}\right]^{T}}$ and $y$ are input vectors and output value of FIS, respectively. $A_{i}^{j}(i=1,2, . ., n)$ and $B_{k}(k=1,2, \ldots, m)$ are linguistic variables of fuzzy sets in subspace $U_{i}$ and $V_{k}$ described by their membership functions $\mu_{A_{i}^{j}}\left(x_{i}\right)$ and $\mu_{B_{k}^{j}}\left(y_{k}\right), j=1,2, \ldots, M$. $M$ is total number of the fuzzy rules. By using product inference, singleton-fuzzifier, and center-average defuzzifier strategies, output of FIS can be expressed as

$$
y(x)=\hat{\Omega}(x \mid \hat{\Theta})=\frac{\sum_{j=1}^{M} \bar{y}^{j}\left(\prod_{i=1}^{n} \mu_{A_{i}^{j}}\left(x_{i}\right)\right)}{\sum_{j=1}^{M}\left(\prod_{i=1}^{n} \mu_{A_{i}^{j}}\left(x_{i}\right)\right)}=\hat{\Theta} \xi(x)
$$

where $\mu_{A_{i}^{j}}\left(x_{i}\right)$ is membership function value of fuzzy variable $x_{i}, \bar{y}^{j}$ is the point at which $\mu_{B_{k}^{j}}\left(y_{k}\right)$ achieves its maximum value, and it is assumed that $\mu_{B_{k}^{j}}\left(\bar{y}^{j}\right)=1$.

$$
\hat{\Theta}=\left(\begin{array}{cccc}
\bar{y}_{1}^{1} & \bar{y}_{1}^{2} & \cdots & \bar{y}_{1}^{M} \\
\bar{y}_{2}^{1} & \bar{y}_{2}^{2} & \cdots & \bar{y}_{2}^{M} \\
\vdots & \vdots & \ddots & \vdots \\
\bar{y}_{m}^{1} & \bar{y}_{m}^{2} & \cdots & \bar{y}_{m}^{M}
\end{array}\right)
$$

Eq. (2) is adjustable parameter matrix and $\xi(x)=\left[\xi^{1}(x), \xi^{2}(x), \ldots, \xi^{M}(x)\right]^{T}$ is fuzzy basis function vector, in which $\xi^{j}(x)$ is defined as

$$
\xi^{j}(x)=\frac{\prod_{i=1}^{n} \mu_{A_{i}^{j}}\left(x_{i}\right)}{\sum_{j=1}^{M} \prod_{i=1}^{n} \mu_{A_{i}^{j}}\left(x_{i}\right)}(j=1,2, \ldots, M)
$$

From analysis of the FIS, we must give fuzzy rules of mass point clouds, and then normal of point clouds can be estimated for different kind.

\subsection{Description of Fuzzy Rules}

The value of $\left[x_{k n n}, x_{c u r}\right]$ can express the density and sharp distribution of point clouds. The combinations of the two input variables with different values are inputted into fuzzy system and compared with the fuzzy rules. Which part of dental point clouds belong to can be judged by fuzzy inference engine. The fuzzy rules are expressed as follows:

$\mathrm{R}_{1}$ : if $x_{k n n}$ is small and $\left|x_{\text {cur }}\right|$ is small, then $y$ belongs to class one;

$\mathrm{R}_{2}$ : if $x_{k n n}$ is small and $\left|x_{\text {cur }}\right|$ is large, then $y$ belongs to class two;

$\mathrm{R}_{3}$ : if $x_{k n n}$ is large and $\left|x_{c u r}\right|$ is large, then $y$ belongs to class three ;

where "small" and "large" in the rules are linguistic values of fuzzy sets in subspace 
$U_{i}$ described by their membership functions $\mu_{A_{i}^{j}}\left(x_{i}\right), j=1,2, \ldots, M . M$ is total number of the fuzzy rules. For convenience, triangular-type membership functions for $x_{k n n}$ and $x_{c u r}$ are selected in this system in Fig. 4.
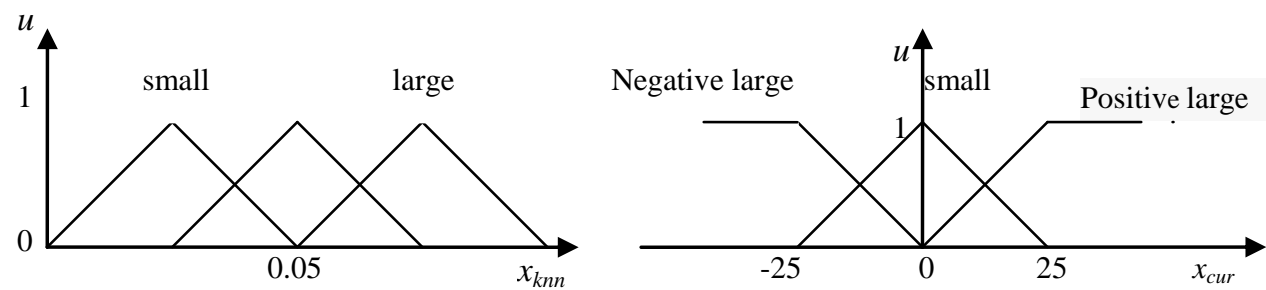

Figure 4. Triangular-type Membership Functions for $x_{k n n}$ and $x_{c u r}$

In the specific rules, $\left|{ }^{x_{c u r}^{i-1}}\right|$ and $\left.\right|^{x_{c u r}^{i+1}} \mid$ is two points in the curvature of the values before and after data point x. $x_{k n n}$ large point cloud data distribution is relatively loose, whereas that data point distribution density. $\left|x_{\text {cur }}\right|$ large illustrate that point clouds location curvature change is very big and point clouds is smooth. If the point clouds distribute loose and changes small curvature, then illustrate the local area is smooth. If point cloud distribute density and changes large curvature, then show the local normal vector will be a special change and local area may be contains chip features or sharp. It needs more changes the curvature of the point clouds and what kinds of characteristics near the concrete need to determine. If the point clouds at a $\left.\right|^{x_{c u r}^{i-1}} \mid$ and $\left.\right|^{x_{c u r}^{i+1}} \mid$ is small, then the local area contains chip characteristic. If curvature of the point clouds change $\left.\right|^{x_{c u r}^{i-1}} \mid$ and $\mid x_{\text {cur }}^{i+1}$ have big or small, then the local area with sharp features. To system calculation efficiency, triangular membership functions are adopted for membership function of $x_{k n n}$ and $x_{c u r}$. The input of fuzzy rules is obtained by the experiment of $x_{k n n}$ and $x_{c u r}$ corresponding "small", "large".

\section{Experimental Results}

The purpose of the experiments is to evaluate the performance of this proposed method by irregular models of denture. Experimental results are implemented on a PC equipped with AMD phenom(tm)IIX4 B95, 2.99GHz CPU, 3G main memory. And point clouds are from 3D laser scanner, PICZA LPX-250.

We can classify point clouds by the given algorithm and then fit the surface by implicit function. The point clouds are divided into sub-domains by octree structure firstly in Fig. 5 . Then the $k \mathrm{NN}$ of each point is computed and connected the points in sub-domain, and therefore we can calculate the curvature. Here $k=5$ and the value of curvature is regarded as small if $x_{c u r} \in[-25,25]$ and as large if $x_{c u r}$ beyond the scope through this experiment. Next, input $x_{k n n}, x_{c u r}$ into FIS and infer to the value of $y$. The corresponding normal estimation method is determined according to the value of $y$. At last, the models of denture are reconstructed using implicit function in Fig. 7 while the reconstruction surface did not classified in Fig.6. 


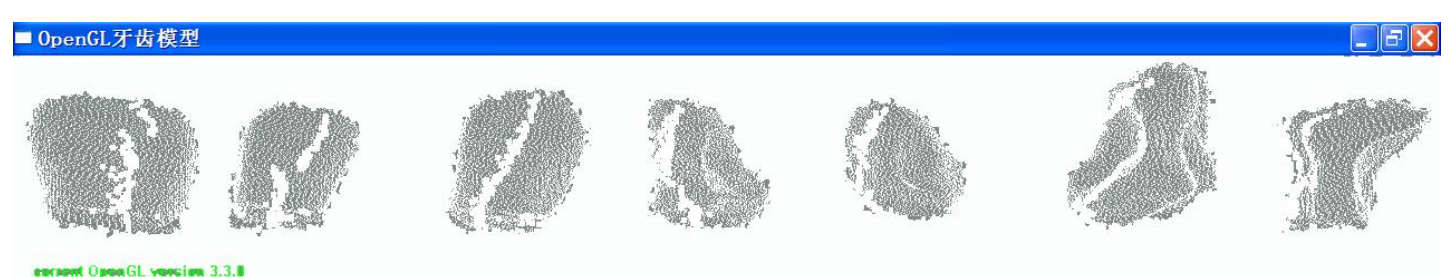

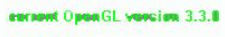

FPS 59.17

$\times 0.00 \times 1.0020 .08$

(a) seven teeth on the right

Made In 2012

E OpenGL牙齿模型

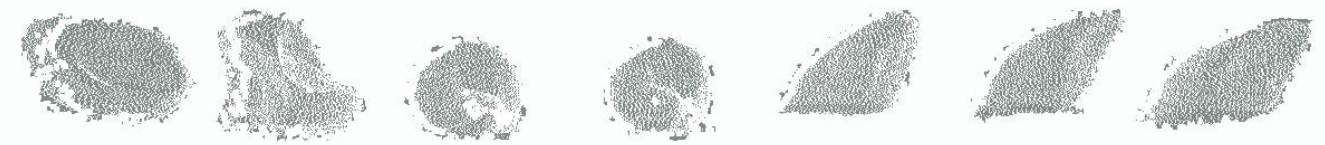

current $\bullet$ penGL versien 3.3.4

FPS 59.17

$\times 1.80$ y 1.0120 .01

(b) seven teeth on the left

Made In 2012

Figure 5. Point Clouds of Denture

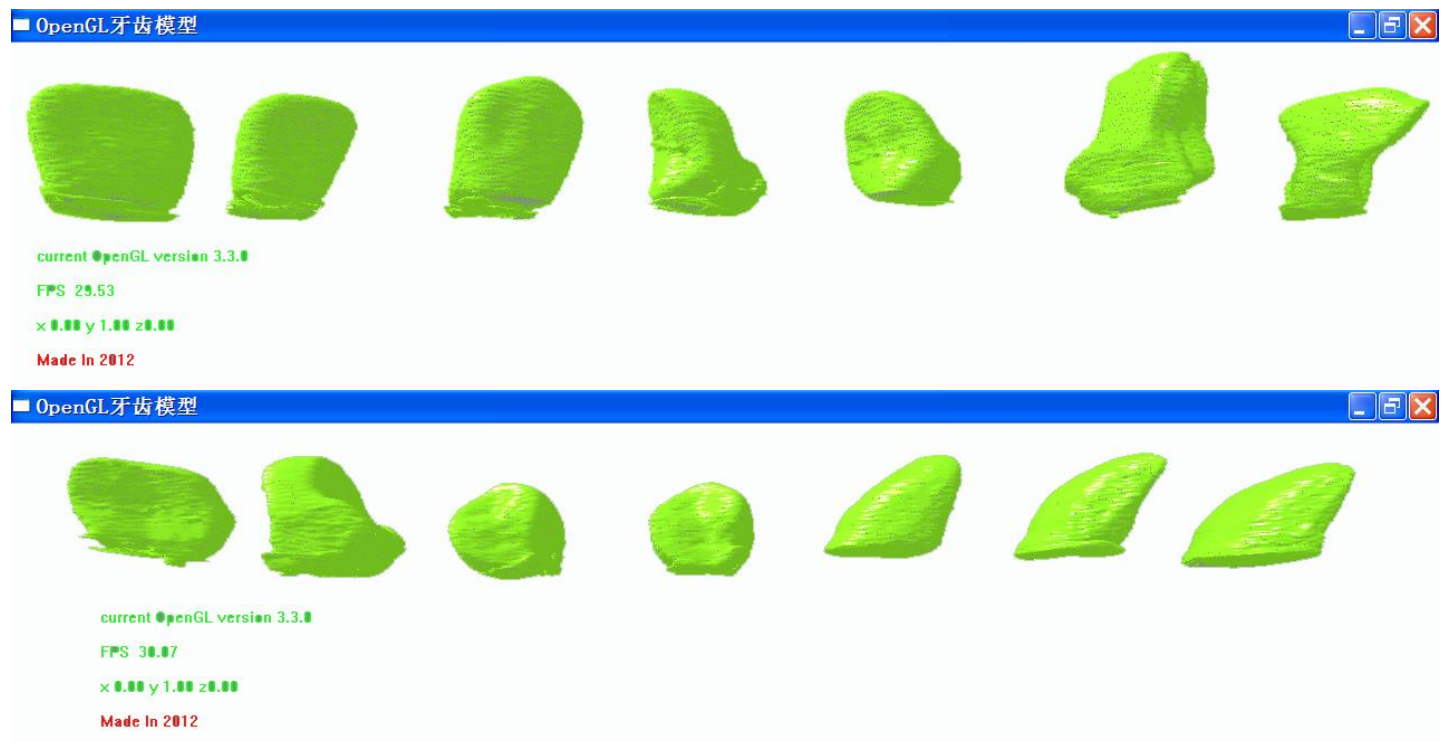

Figure 6. Reconstruction Result without Classification

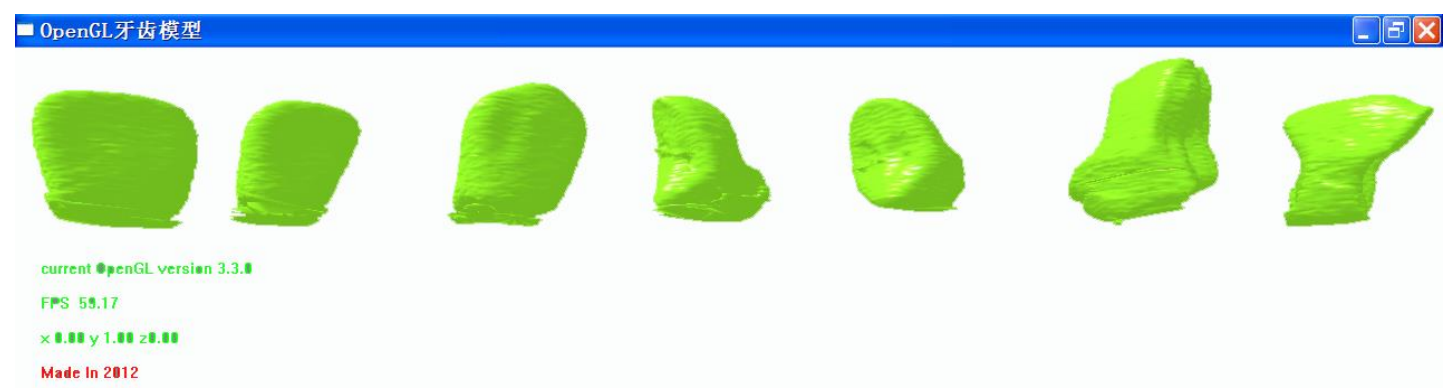




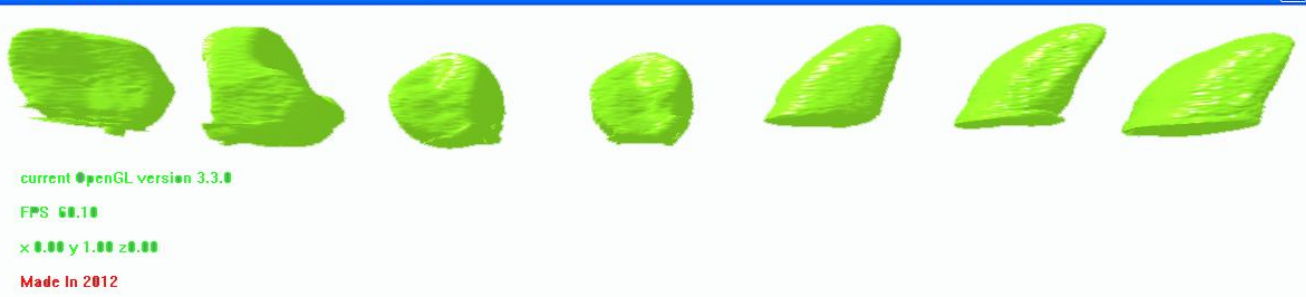

Figure 7. Reconstruction Result after Classification

\section{Conclusions}

The irregular model is complex object that some parts are smooth and some parts are irregular including sharp features. In order to improve the precision, a fuzzy classification strategy algorithm is proposed. The $k \mathrm{NN}$ of mass point clouds and curvature in the subdomain are input into FIS, and the output of FIS can determine which part of point clouds belong to. In this way, the point clouds in the smooth area are constructed by implicit function directly and ones in other regions of thin or sharp area are reconstructed by attach points. This method is simpler than those complex methods used on the whole point clouds directly. Further work concerns a universal fuzzy inference algorithm applying to other complex model.

\section{Acknowledgments}

This work was jointly supported by the National Natural Science Foundation of China (Grant No. 61403222), Natural Science Foundation of Heilongjiang Province (Grant No. F201439, F201440,)

\section{References}

[1] F. Hetroy, S. Rey and C. Andujar, "Mesh repair with user-friendly topology control", Computer Aided Design, vol. 43, no. 1, (2011), pp. 101-113.

[2] F. Schindler, W. Worstner and J. M. Frahm, "Classification and reconstruction of surfaces from point clouds of man-made objects", Computer Vision Workshops (ICCV Workshops), (2011).

[3] M. Attene, M. Campen and L. Kobbelt, "Polygon mesh repairing: an application perspective", ACM Computing Surveys (CSUR), vol. 45, no. 2, (2013), pp. 542-548.

[4] Y. L. Chen and S. H. Lai, "An orientation inference framework for surface reconstruction from unorganized point clouds", IEEE Transactions on Image Processing, no. 20, (2011), pp. 762-775.

[5] J. Wang, Z. W. Yang and F. L. Chen, "A variational model for normal computation of point clouds", The Visual Computer, vol. 28, no. 2, (2012), pp. 163-174.

[6] G. H. Bendels, R. Schanbel and R. Klein, "Detail-preserving surface inpainting", 6th International Symposium on Virtual Reality, Archaeology and Cultural Heritage, Pisa: Eurogrphics Association, (2011).

[7] L. S. Tekumalla and E. A. Cohen, "A hole-filling algortithm for triangular meshes, Technical Report UUCS-04-019", School of Computing, Univ. of Utah, (2004).

[8] Y. J. Liu and Y. D. Zhang, "A novel normal estimation based on fuzzy inference for mass point clouds of denture in the three-dimensional reconstruction", ICIC Express Letters Part B: applications, vol. 3, no. 4, (2012), pp. 1-7.

[9] M. V. J. Parus and I. Kolingerova, "Simple holes triangulation in surface reconstruction", Proceedings of Algorithm, (2011); Podbanske, Solvakia.

[10] J. C. Carr, R. K. Beatson and J. B. Cherrie, "Reconstruction and representation of 3D objects with radial basis functions", 28th annual conference on Computer graphics and interactive techniques (SIGGRAPH), (2009), pp. 67-76.

[11] N. Amenta and Y. J. Kilk, "Defining point-set surfaces", ACM Transactions on Graphics, vol. 23, no. 3, (2004), pp. 264-270.

[12] P. Liepa, "Filling holes in meshes", Proceedings of the Eurographics/ ACM Siggraph Symposium on Processing, Aachen, Germany, (2013).

[13] H. Przybilla, "Fusion of terrestrial lasers canning and digital photogrammetry", IEVM, (2005)

[14] S. Xu, A. Georghiades and H. Rushmeier, "Image guided geometry inference", 3rd International Symposium on 3D Data Processing, Visualization and Transmission, (2006); Washington, USA. 
International Journal of $u-$ and e- Service, Science and Technology

Vol.9, No. 1 (2016)

[15] D. Matteo, V. Andrea and S. Roberto, "Image guided reconstruction of un-sampled data: a filling technique for cultural heritage models", International Journal Comput Vis, vol. 94, (2011), pp. 2-11.

[16] W. Zhao, S. M. Gao and H. W. Lin, "A robust hole-filling algorithm for triangular mesh", Visual Comput, vol. 23, (2007), pp. 987-997. 